\title{
Entanglement sharing with separable states
}

\author{
Ladislav Mišta Jr. ${ }^{1}$ \\ ${ }^{1}$ Department of Optics, Palacký University, 17. listopadu 12, 77146 Olomouc, Czech Republic
}

(Dated: September 17, 2018)

\begin{abstract}
We propose an entanglement sharing protocol based on separable states. Initially, two parties, Alice and Bob, share a two-mode separable Gaussian state. Alice then splits her mode into two separable modes and distributes them between two players. Bob is separable from the players but he can create entanglement with either of the players if the other player moves to his location and collaborates with him. Any two parties are separable and the creation of entanglement is thus mediated by transmission of a mode which is separable from individual modes on Alice's and Bob's side. For the state shared by the players and Bob one cannot establish entanglement between any two modes even with the help of operation on the third mode provided that Bob is restricted to Gaussian measurements and the state thus carries a nontrivial signature of bound entanglement. The present protocol also demonstrates switching between different separability classes of tripartite systems by coherent operations on its bipartite parts and complements studies on protocols utilizing mixed partially entangled multipartite states.
\end{abstract}

\section{INTRODUCTION}

Three correlated elementary quantum systems represent a basic primitive which already may exhibit genuine multipartite phenomena. The discovery that tripartite entanglement can provide a stronger violation of local realism [1, 2] than bipartite one has triggered a large research activity with the aim to characterize it and find its applications. Early studies of tripartite entanglement focused on systems of three two-level particles (qubits) for which new forms of multipartite bound entanglement [3, 4] and inequivalent entanglement classes [5] have been found. Three qubits also proved to be a suitable platform for construction of a classical analog of bound entanglement know as bound information [6], which so far has not been found in the bipartite scenario. In comparison with two qubits which can only be separable or entangled three-qubit states can be divided into five separability classes [7] in dependence on their separability properties with respect to different qubits. Most of the applications of the three-qubit entanglement rely on the utilization of pure states from the class of fully inseparable states which are entangled with respect to all three qubits. They involve various protocols for information splitting ranging from secret sharing [8], telecloning [9] and assisted teleportation [10] as well as protocols for construction of quantum gates [11] or controlled quantum cryptography [12].

Tripartite entanglement has also been investigated within the framework of Gaussian states [13] of infinitelydimensional quantum systems. A convenient prototype of such a system is a system of three modes $A, A^{\prime}$ and $B$ of an electromagnetic field which are characterized by position $x_{j}$ and momentum $p_{j}$ quadrature operators satisfying the canonical commutation rules $\left[x_{j}, p_{k}\right]=i \delta_{j k}$, $j, k=A, A^{\prime}, B$. Quantum states of three modes can be represented in phase space by a 6 -variate Wigner quasiprobability distribution [14] and the set of Gaussian states comprises states with a Gaussian Wigner function. A three-mode Gaussian state $\rho_{A A^{\prime} B}$ is therefore fully characterized by the vector of coherent displacements $d=\langle\xi\rangle=\operatorname{Tr}\left(\rho_{A A^{\prime} B} \xi\right)$, where we have introduced a column vector $\xi=\left(x_{A}, p_{A}, x_{A^{\prime}}, p_{A^{\prime}}, x_{B}, p_{B}\right)^{T}$, and by a $6 \times 6$ covariance matrix $(\mathrm{CM})$ with elements $\gamma_{i j}=\left\langle\left\{\xi_{i}-d_{i}, \xi_{j}-d_{j}\right\}\right\rangle, i, j=1, \ldots, 6$, where $\{A, B\}=$ $A B+B A$ is the anticommutator.

Following the classification of Ref. [7] we can divide three-mode states into five separability classes involving [15]:

1. Fully inseparable states which are entangled with respect to all three bipartite splittings of modes $A, A^{\prime}$ and $B$ into two groups. That is states entangled across $A-\left(A^{\prime} B\right), A^{\prime}-(A B)$ as well as $B-\left(A A^{\prime}\right)$ splitting.

2. One-mode biseparable states which are entangled with respect to two bipartite splittings, but separable with respect to the third one. Such a state exhibits entanglement across, e.g, $A-\left(A^{\prime} B\right)$ and $A^{\prime}-(A B)$ splitting but it is separable with respect to $B-\left(A A^{\prime}\right)$ splitting.

3. Two-mode biseparable states which are entangled across one bipartite splitting, but separable with respect to the remaining two splittings. The state is therefore entangled, e.g., across $A-\left(A^{\prime} B\right)$ splitting but separable with respect to $A^{\prime}-(A B)$ and $B-\left(A A^{\prime}\right)$ splittings.

4. Three-mode biseparable states which are separable across all three bipartite splittings but which cannot be written as a convex mixture of triple product states.

5. Fully separable states which can be written as a convex mixture of triple product states.

Like in the qubit case a genuine tripartite entanglement carried by fully inseparable three-mode states is practically exclusively used as a resource in quantum information protocols. It is due to a relative ease of its preparation [16, 17], detection [18] and a number of quantum protocols which this type of entanglement offers [19 21]. On the other hand, the other classes carry only partial or no entanglement, some exist just in the mixed-state scenario (two-mode and three-mode biseparable states [22]) and one might be then tempted to doubt about their practical utility. However, the astonishing protocol for entanglement distribution by a separable system [23] 
teaches us about the opposite. Originally developed for qubits [23], later extended to Gaussian states [24], and experimentally demonstrated in [25], it shows that also other, even mixed and just partially separable states may demonstrate new phenomena which are not encountered in the context of fully inseparable states. It demonstrates that two distant observers, Alice and Bob, can entangle modes $A$ and $B$ held by them by sending a third separable mode $A^{\prime}$ between them. Initially, Alice holds modes $A$ and $A^{\prime}$ whereas Bob holds mode $B$ of a suitable fully separable Gaussian state. By a beam splitter on modes $A$ and $A^{\prime}$ Alice then transforms the state to the state entangled only across $A-\left(A^{\prime} B\right)$ splitting (two-mode biseparable state) and transmits the separable mode $A^{\prime}$ to Bob. He finally superimposes the received mode $A^{\prime}$ with his mode $B$ on another beam splitter and thus he entangles modes $A$ and $B$ whereas mode $A^{\prime}$ still remains separable from the two-mode subsystem $(A B)$ (one-mode biseparable state). The protocol thus shows that direct transmission of entanglement is not necessary to entangle two separate parties but only communication of a separable quantum system, local operations and a priori shared suitable fully separable state of three quantum systems suffice to accomplish the task.

In this paper we demonstrate the utility of mixed partially separable states from other separability classes for implementation of a certain entanglement sharing scheme. Specifically, we show that there is a correlated separable Gaussian state of two possibly distant modes $A$ and $B$ such that if mode $A$ is split into two modes $A$ and $A^{\prime}$ this creates entanglement of each of the modes with the distant mode $B$ and the other split mode taken together. More precisely, the splitting entangles mode $A$ with the pair of modes $\left(A^{\prime} B\right)$ and mode $A^{\prime}$ with the pair of modes $(A B)$ at the same time. Moreover, any two modes are separable in the prepared three-mode state. By transmitting the mode $A^{\prime}(A)$ which is separable from the mode $A\left(A^{\prime}\right)$ to the location of mode $B$ the entanglement can be transformed by a simple beam splitter into entanglement of mode $A\left(A^{\prime}\right)$ and the distant mode $B$. The protocol thus shares similarity with the quantum secret sharing protocol [8, 26] for entanglement [27] in which a dealer splits one part of a bipartite entanglement among several players in such a way that some collections of players can recover the entanglement with the dealer whereas the other collections cannot. If in the present case the modes $A$ and $B$ of the initial separable state are shared by two observers, called Alice and Bob, then entanglement created by splitting on Alice's side of mode $A$ into two modes $A$ and $A^{\prime}$ can be turned into the entanglement between Alice and Bob only if Bob has physically at his disposal also either of the split modes $A$ or $A^{\prime}$. Remarkably, there is a certain interval of entanglement strengths for which Bob can establish entanglement with Alice by the coherent beam splitting operation on his mode and the received mode but he cannot establish it by any Gaussian measurement on his mode $B$ followed by displacement of modes $A$ and $A^{\prime}$ which

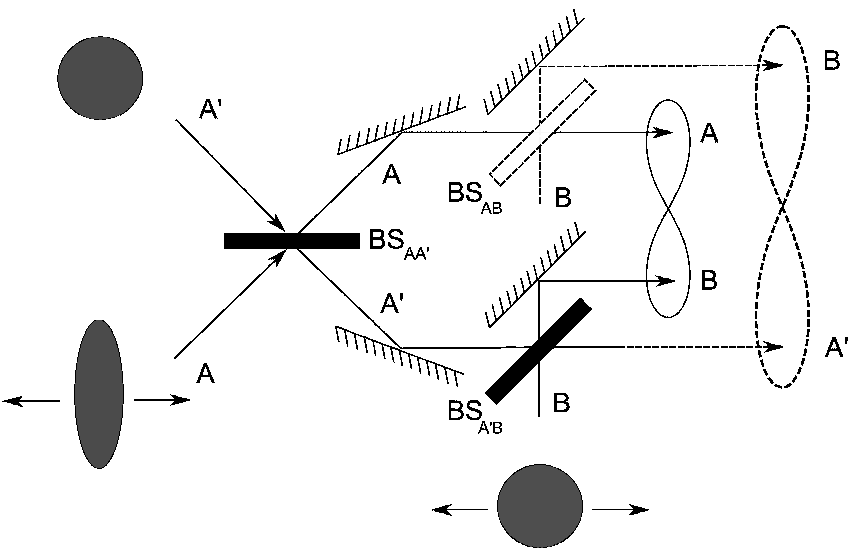

FIG. 1: Scheme of the entanglement sharing protocol. Mode $A$ in a position squeezed vacuum state and a vacuum mode $B$ are displaced as in Eq. (2). Mode $A$ is then split on a balanced beam splitter $B S_{A A^{\prime}}$ into two modes $A$ and $A^{\prime}$ which creates a state with no two-mode entanglement which possesses entanglement across $A-\left(A^{\prime} B\right)$ and $A^{\prime}-(A B)$ splittings and which is separable across $B-\left(A A^{\prime}\right)$ splitting. If modes $A^{\prime}$ and $B$ are superimposed on a balanced beam splitter $B S_{A^{\prime} B}$ and the dashed beam splitter $B S_{A B}$ is absent, entanglement is localized between modes $A$ and $B$ (solid lines with arrows). If instead the beam splitter $B S_{A^{\prime} B}$ is removed and modes $A$ and $B$ are superimposed on a dashed balanced beam splitter $B S_{A B}$ entanglement is localized between modes $A^{\prime}$ and $B$ (dashed lines with arrows). In both cases the resulting state is entangled across all three bipartite splittings and therefore the state is genuinely tripartite entangled. See text for details.

challenges the question about the presence of bound entanglement in the considered state. Like in the case of the entanglement distribution by a separable ancilla the protocol works only with mixed states and starts with a suitable fully separable three-mode Gaussian state. In contrast with a two-mode biseparable state which is created in the second step of the entanglement distribution protocol a one-mode biseparable state is created in the intermediate step of the present entanglement sharing protocol. Likewise, a one-mode biseparable state appears at the final step of the entanglement distribution protocol whereas the entanglement sharing scheme is crowned by a fully inseparable state.

The paper is structured as follows. In Sec II we explain the entanglement sharing protocol. In Sec. [II] we show the gap between unitary and measurement-based localizability of the entanglement for the state from the intermediate step of the protocol. Sec. IV contains discussion and conclusion.

\section{ENTANGLEMENT SHARING PROTOCOL}

The scheme of the protocol is depicted in Fig. 1 The protocol starts with a Gaussian state of two modes $A$ and $B$ shared by a sender Alice and a receiver Bob which has 
the covariance matrix (CM) of the form:

$$
\gamma_{A B}=\left(\begin{array}{cccc}
1+e^{-2 r}\left(e^{2 \varepsilon}-1\right) & 0 & e^{-2 r}-1 & 0 \\
0 & e^{2 r} & 0 & 0 \\
e^{-2 r}-1 & 0 & 2-e^{-2 r} & 0 \\
0 & 0 & 0 & 1
\end{array}\right),
$$

where $r \geq 0$ is the squeezing parameter and $\varepsilon \geq 0$ is a noise parameter which will be specified later. The right upper $2 \times 2$ off-diagonal block is a diagonal matrix $\operatorname{diag}\left(e^{-2 r}-1,0\right)$ with zero determinant and hence the state is separable 28]. The state can be easily prepared by displacing position quadratures of the state in mode $A$ with the $\mathrm{CM} \gamma_{A}=\operatorname{diag}\left(e^{-2(r-\varepsilon)}, e^{2 r}\right)$ and the vacuum mode $B$ with $\mathrm{CM} \gamma_{B}=\mathbb{1}$ as

$$
x_{A} \rightarrow x_{A}+\bar{x}, \quad x_{B} \rightarrow x_{B}-\bar{x} .
$$

Here, $\bar{x}$ is the classical Gaussian distributed displacement with variance satisfying $\left\langle\bar{x}^{2}\right\rangle=\left(1-e^{-2 r}\right) / 2$ and $\mathbb{1}$ is the $2 \times 2$ identity matrix.

Next, Alice splits her mode $A$ into two modes $A$ and $A^{\prime}$ by superimposing mode $A$ with vacuum mode $A^{\prime}$ on a balanced beam splitter. If we denote the vacuum CM of mode $A^{\prime}$ as $\gamma_{A^{\prime}}=\mathbb{1}$ and describe the beam splitter by the orthogonal matrix

$$
U_{i j}=\frac{1}{\sqrt{2}}\left(\begin{array}{cc}
\mathbb{1} & \mathbb{1} \\
\mathbb{1} & -\mathbb{1}
\end{array}\right),
$$

where $i=A, j=A^{\prime}$, we get the three-mode Gaussian state with the following CM

$$
\gamma_{A A^{\prime} B}=\left(\begin{array}{ccc}
\alpha & \delta & \tau \\
\delta & \alpha & \tau \\
\tau & \tau & \beta
\end{array}\right)
$$

with $\alpha, \beta, \tau$ and $\delta$ being diagonal matrices of the form $\alpha=$ $\operatorname{diag}\left(2+e^{-2 r}\left(e^{2 \varepsilon}-1\right), e^{2 r}+1\right) / 2, \beta=\operatorname{diag}\left(2-e^{-2 r}, 1\right)$, $\tau=\operatorname{diag}\left(e^{-2 r}-1,0\right) / \sqrt{2}$ and $\delta=\operatorname{diag}\left(e^{-2 r}\left(e^{2 \varepsilon}-1\right), e^{2 r}-\right.$ $1) / 2$.

The performance of the proposed protocol is enabled by the remarkable separability properties of the state with CM (4). Let us investigate first the separability of the state with respect to the splitting of modes $A, A^{\prime}$ and $B$ into two groups $(1 \times 2$-mode separability). Beam splitting transformation (3) on mode $A$ and mode $A^{\prime}$ cannot create entanglement with mode $B$ and hence the state is separable across the $B-\left(A A^{\prime}\right)$ splitting. The state is, however, entangled with respect to the remaining $A-\left(A^{\prime} B\right)$ and $A^{\prime}-(A B)$ splittings as can be easily proven using the positive partial transposition criterion [29, 30] expressed in terms of symplectic invariants [31]. Specifically, separability of mode $X$ from a pair of modes $(Y Z)$ in a generic Gaussian state of three modes $X, Y$ and $Z$ with the $\mathrm{CM} \gamma$ can be determined from the symplectic invariants of the matrix $\gamma^{\left(T_{x}\right)} \equiv \Lambda_{x} \gamma \Lambda_{x}^{T}$ associated with the partial transpose of the state with respect to mode $X$. Here, $\Lambda_{x} \equiv \sigma_{z}^{(X)} \oplus \mathbb{1}^{(Y)} \oplus \mathbb{1}^{(Z)}$, where $\sigma_{z}=\operatorname{diag}(1,-1)$ is the Pauli diagonal matrix. The matrix $\gamma^{\left(T_{x}\right)}$ possesses three symplectic invariants denoted as $I_{1}, I_{2}$ and $I_{3}=\operatorname{det}(\gamma)$ which coincide with the coefficients of the characteristic polynomial of the matrix $\Omega \gamma^{\left(T_{x}\right)}$, i.e.

$$
\operatorname{det}\left(\Omega \gamma^{\left(T_{x}\right)}-q \mathbb{1}\right)=q^{6}+I_{1} q^{4}+I_{2} q^{2}+I_{3},
$$

where

$$
\Omega=\bigoplus_{i=1}^{3}\left(\begin{array}{cc}
0 & 1 \\
-1 & 0
\end{array}\right)
$$

According to the criterion 31] mode $X$ is entangled with the two-mode subsystem $(Y Z)$ if

$$
\Sigma_{x}=I_{3}-I_{2}+I_{1}-1<0 .
$$

In the case of the CM (4) one gets explicitly after some algebra that

$$
\Sigma_{A}=8 e^{\varepsilon-r} \sinh (\varepsilon-r) \sinh ^{2}(r),
$$

which implies that if $r>\varepsilon$ it holds that $\Sigma_{A}<0$ and there is entanglement across $A-\left(A^{\prime} B\right)$ splitting. Owing to the symmetry of the state under the exchange of modes $A$ and $A^{\prime}$ (bisymmetric state [32]) it follows immediately that $\Sigma_{A}=\Sigma_{A^{\prime}}$ and the state is therefore also entangled across $A^{\prime}-(A B)$ splitting. Thus, the studied state is separable across one bipartite splitting and therefore it belongs to the class of one-mode biseparable Gaussian states.

Let us focus now on the separability of the two-mode reductions $(1 \times 1$-mode separability) of the state with the CM (44). Due to the separability of the $B-\left(A A^{\prime}\right)$ splitting mode $B$ is inevitably separable both from the mode $A$ as well as from the mode $A^{\prime}$. The reduced state of modes $A$ and $A^{\prime}$ was created by mixing of the state with $\mathrm{CM}$ $\gamma_{A}=\operatorname{diag}\left[1+e^{-2 r}\left(e^{2 \varepsilon}-1\right), e^{2 r}\right]$ with the vacuum state on a beam splitter (3). Both the eigenvalues of the CM $\gamma_{A}$ are lower bounded by the unity and hence the corresponding normally ordered $\mathrm{CM} \gamma_{A}^{(\mathcal{N})} \equiv \gamma_{A}-\mathbb{1}$ is positive semidefinite. The normally ordered characteristic function of the state then possesses a Fourier transform which is not more singular than a Dirac delta function and the state with the $\mathrm{CM} \gamma_{A}$ is thus classical. As mixing of such a state with a vacuum state on a beam splitter cannot create entanglement [33] mode $A$ is therefore separable from mode $A^{\prime}$ in the state with CM (4). In summary, for the state with the CM (4) any two modes are separable.

Note, that the aforementioned separability properties of the state with CM (4) can exist only in a mixed-state scenario. Indeed, for a pure state separability of mode $B$ from modes $\left(A A^{\prime}\right)$ implies the state to be a product state across the $B-\left(A A^{\prime}\right)$ splitting. Likewise, the separability of the mode $A$ from the mode $A^{\prime}$ implies that the reduced state of the modes $A$ and $A^{\prime}$ is also a product state. Consequently, a pure three-mode state where the mode $B$ is separable from modes $\left(A A^{\prime}\right)$ and mode $A$ 
is at the same time separable from mode $A^{\prime}$ is therefore a triple product state which is fully separable. Such a state, however, cannot possess entanglement across, e.g., $A-\left(A^{\prime} B\right)$ splitting as is the case of our state.

At the final stage of the protocol Alice keeps mode $A$ and sends the separable mode $A^{\prime}$ to Bob. He superimposes the mode with his mode $B$ on a balanced beam splitter $U_{B A^{\prime}}$ given in Eq. (3) where $i=B$ and $j=A^{\prime}$ which creates a state with $\mathrm{CM}$

$$
\tilde{\gamma}_{A A^{\prime} B}=\left(\begin{array}{ccc}
\alpha & \frac{\tau-\delta}{\sqrt{2}} & \frac{\tau+\delta}{\sqrt{2}} \\
\frac{\tau-\delta}{\sqrt{2}} & \frac{\alpha+\beta-2 \tau}{2} & \frac{\beta-\alpha}{2} \\
\frac{\tau+\delta}{\sqrt{2}} & \frac{\beta-\alpha}{2} & \frac{\alpha+\beta+2 \tau}{2}
\end{array}\right)
$$

where the $2 \times 2$ submatrices $\alpha, \beta, \tau$ and $\delta$ are given below Eq. (4). If, on the other hand, Alice keeps mode $A^{\prime}$ and sends the separable mode $A$ to Bob who mixes it with his mode on the beam splitter

$$
U_{A B}=\frac{1}{\sqrt{2}}\left(\begin{array}{cc}
\mathbb{1} & -\mathbb{1} \\
\mathbb{1} & \mathbb{1}
\end{array}\right)
$$

the $\mathrm{CM}$ of the resulting state reads

$$
\tilde{\tilde{\gamma}}_{A A^{\prime} B}=\left(\begin{array}{ccc}
\frac{\alpha+\beta-2 \tau}{2} & \frac{\delta-\tau}{\sqrt{2}} & \frac{\alpha-\beta}{2} \\
\frac{\delta-\tau}{\sqrt{2}} & \alpha & \frac{\delta+\tau}{\sqrt{2}} \\
\frac{\alpha-\beta}{2} & \frac{\delta+\tau}{\sqrt{2}} & \frac{\alpha+\beta+2 \tau}{2}
\end{array}\right)
$$

By retaining only the modes $A\left(A^{\prime}\right)$ and $B$ it then follows that Alice and Bob are left with a reduced twomode state with the $\mathrm{CM}$

$$
\tilde{\gamma}_{A B}=\tilde{\tilde{\gamma}}_{A^{\prime} B}=\left(\begin{array}{cc}
\alpha & \frac{\delta+\tau}{\sqrt{2}} \\
\frac{\delta+\tau}{\sqrt{2}} & \frac{\alpha+\beta+2 \tau}{2}
\end{array}\right) \text {, }
$$

which describes an entangled state provided that the squeezing parameter $r$ is large enough. The threshold squeezing $r_{\mathrm{e}}$ above which the entanglement appears can be derived from the two-mode version of the sufficient condition for entanglement 31]

$$
\operatorname{det} \tilde{\gamma}_{A B}-\Delta+1<0
$$

where $\Delta=\operatorname{det} \alpha+\frac{1}{4} \operatorname{det}(\alpha+\beta+2 \tau)-\operatorname{det}(\delta+\tau)$. Substituting here for the matrices $\alpha, \beta, \tau$ and $\delta$ defined below Eq. (4) one finds, that mode $A\left(A^{\prime}\right)$ is entangled with mode $B$ if the squeezing $r$ satisfies $r>r_{\mathrm{e}}$, where

$$
r_{\mathrm{e}}=\frac{1}{2} \ln \left[\frac{11 e^{2 \varepsilon}+8 \sqrt{2}-13+\sqrt{\left(11 e^{2 \varepsilon}+8 \sqrt{2}-13\right)^{2}+4 e^{2 \varepsilon}(8 \sqrt{2}-1)}}{2(8 \sqrt{2}-1)}\right]
$$

It is further of interest to look at the $1 \times 2$-mode separability of the states with CMs (9) and (11) from the last step of the protocol. We have already seen that for the state with the CM (9) [(11)] mode $A\left(A^{\prime}\right)$ is entangled with mode $B$. This implies, that there is entanglement across $A-\left(A^{\prime} B\right)\left[A^{\prime}-(A B)\right]$ as well as $B-\left(A A^{\prime}\right)$ $\left[B-\left(A A^{\prime}\right)\right]$ splitting. But what about entanglement across the remaining $A^{\prime}-(A B)\left[A-\left(A^{\prime} B\right)\right]$ splitting? Analyzing the entanglement using again the criterion (7) one gets for the CMs (9) and (11) the following expressions:

$$
\tilde{\Sigma}_{A^{\prime}}=\tilde{\tilde{\Sigma}}_{A}=\frac{\Sigma_{A}}{4}
$$

where the quantity $\tilde{\Sigma}_{A^{\prime}}\left(\tilde{\tilde{\Sigma}}_{A}\right)$ characterizes separability of the mode $A^{\prime}(A)$ in the state with the CM (9) [(11)] and $\Sigma_{A}$ is given in Eq. (8). Hence, for the considered squeezing $r>\varepsilon$ the state in the last step of the present protocol is in both cases entangled across all three bipartite splittings and it therefore carries a genuine threemode entanglement. The proposed sharing scheme thus also illustrates remarkable transformation properties of the three-mode fully separable state given by a product of a separable state of modes $A$ and $B$ with CM (1) and a vacuum state of mode $A^{\prime}$. Namely, the beam splitting transformation (3) on a two-mode subsystem formed by modes $A$ and $A^{\prime}$ transforms the state into the onemode biseparable state which is separable with respect to $B-\left(A A^{\prime}\right)$ splitting and entangled across $A-\left(A^{\prime} B\right)$ and $A^{\prime}-(A B)$ splittings. Moreover, the second beam splitter on modes $A^{\prime}(A)$ and $B$ preserves the latter entanglement and further creates entanglement also across $B-\left(A A^{\prime}\right)$ splitting, i.e., creates a genuine three-mode entanglement.

\section{UNITARY VERSUS MEASUREMENT-BASED LOCALIZABILITY OF THE INTERMEDIATE ENTANGLEMENT}

It can seem for the first sight that Bob may not need coherent beam splitting operation on his mode $B$ and the received mode $A^{\prime}(A)$ to establish entanglement with $\mathrm{Al}$ ice's mode $A\left(A^{\prime}\right)$. One can argue that the participants 
could first establish entanglement between the transmitted mode $A^{\prime}(A)$ (held by Bob) and Alice's mode $A\left(A^{\prime}\right)$ simply by optimally measuring mode $B$ (which is separable from the pair of modes $\left(A A^{\prime}\right)$ ) followed by an optimal displacement of modes $A$ and $A^{\prime}$. The entanglement thus obtained could be subsequently transformed into the entanglement of mode $B$ with Alice's mode $A\left(A^{\prime}\right)$ just by swapping the transmitted mode $A^{\prime}(A)$ with the mode $B$. Now we show, that if Bob is restricted to Gaussian measurements there is a region of squeezing parameters $r$ for which he is unable to establish any entanglement with Alice by this measure-and-displace strategy. The task to be solved can be formulated as a task for finding maximum entanglement which can be localized between modes $A$ and $A^{\prime}$ of the state with CM (4) by optimal Gaussian measurement on mode $B$ of the state. This problem, known as Gaussian localizable entanglement, has already been solved in the literature [34]. It can be conveniently analyzed using the concept of lower symplectic eigenvalue of the partially transposed state, which is for a generic CM $\sigma_{A B}$ of two modes $A$ and $B$ written in the $2 \times 2$-block form with respect to $A-B$ splitting

$$
\sigma_{A B}=\left(\begin{array}{cc}
A & C \\
C^{T} & B
\end{array}\right)
$$

given by 35]

$$
\mu=\sqrt{\frac{\Delta-\sqrt{\Delta^{2}-4 \operatorname{det} \sigma_{A B}}}{2}}
$$

where $\Delta=\operatorname{det} A+\operatorname{det} B-2 \operatorname{det} C$. The state with $\mathrm{CM} \sigma_{A B}$ contains entanglement if and only if $\mu<1$. The symplectic eigenvalue (17) also characterizes the amount of entanglement in the state which can be quantified by the logarithmic negativity $E_{\mathcal{N}}\left(\sigma_{A B}\right)=\max \left(0,-\log _{2} \mu\right)$ [35]. As $E_{\mathcal{N}}$ is a monotonically decreasing function of the symplectic eigenvalue it follows that the smaller the value of $\mu$ the larger the entanglement. For certain families of three-mode Gaussian states one can find even analytically the symplectic eigenvalue (17) for a two-mode state obtained by Gaussian measurement on the third mode minimized over all Gaussian measurements on the mode [34]. The present state with CM (4) belongs to the class of the bisymmetric states for which the problem of the Gaussian localizable entanglement can also be fully resolved using the analytical tools [36]. It turns, that the localizable entanglement between modes $A$ and $A^{\prime}$ is always achieved by homodyne detection of position quadrature $x_{B}$ on mode $B$, i.e., by projection of the mode onto an infinitely squeezed position eigenstate. This gives the minimal lower symplectic eigenvalue (17) of the partial transpose of the conditional state of modes $A$ and $A^{\prime}$ in the form:

$$
\mu_{\mathrm{m}}= \begin{cases}e^{r} & \text { if } r<r_{1} \equiv \frac{1}{2} \ln \left\{\frac{1}{3}+2 \sqrt{-\frac{p}{3}} \cos \left[\frac{1}{3} \arccos \left(-\frac{q}{2} \sqrt{-\frac{27}{p^{3}}}\right)\right]\right\} \\ \sqrt{1+e^{-2 r}\left(e^{2 \varepsilon}-1\right)-\frac{\left(e^{-2 r}-1\right)^{2}}{2-e^{-2 r}}} & \text { otherwise }\end{cases}
$$

where

$$
p=\frac{1}{6}-e^{2 \varepsilon}, \quad q=\frac{5}{54}+\frac{e^{2 \varepsilon}}{6}
$$

Clearly, the condition on the squeezing $r$ for which Bob can localize entanglement between modes $A$ and $A^{\prime}$ can be derived by solving the inequality $\mu_{\mathrm{m}}<1$ with respect to $r$. This gives explicitly that the localizable entanglement is nonzero if $r>r_{\mathrm{m}}$, where

$$
r_{\mathrm{m}}=\frac{1}{2} \ln \left[e^{2 \varepsilon}+\sqrt{e^{2 \varepsilon}\left(e^{2 \varepsilon}-1\right)}\right] .
$$

The performance of our protocol can be illustrated on a particular example when we set $\varepsilon=0.1$, which is depicted in Fig. 2, It follows from Eq. (8) that the state with the CM (4) contains entanglement across $A-\left(A^{\prime} B\right)$ as well as $A^{\prime}-(A B)$ splittings if $r>\varepsilon=0.1$. The entanglement can be transformed into two-mode entanglement of modes $A\left(A^{\prime}\right)$ and $B$ by a balanced beam splitter on modes $A^{\prime}(A)$ and $B$ if $r>r_{\mathrm{e}} \doteq 0.106$. In contrast, Bob can localize some entanglement between modes $A$ and $A^{\prime}$ only if $r>r_{m} \doteq 0.277$ according to Eq. (20). Thus, in the interval $r_{m} \geq r>r_{\mathrm{e}}$ Bob can get entanglement by a coherent operation on his mode and the received mode but cannot create entanglement between Alice's mode and the received mode by any Gaussian measurement on his mode and optimal displacement of Alice's and the received mode. Further analysis shows that the gap between the threshold squeezings (14) and (201) exists also for the other values of the parameter $\varepsilon$. In Fig. 3 we plot the threshold squeezings as well as their difference $r_{\mathrm{m}}-r_{\mathrm{e}}$ as a function of $\varepsilon$. The figure and further calculations reveal that the difference exists for any $\varepsilon>0$ and it is a monotonically increasing function of $\varepsilon$ which asymptotically approaches the limit value 


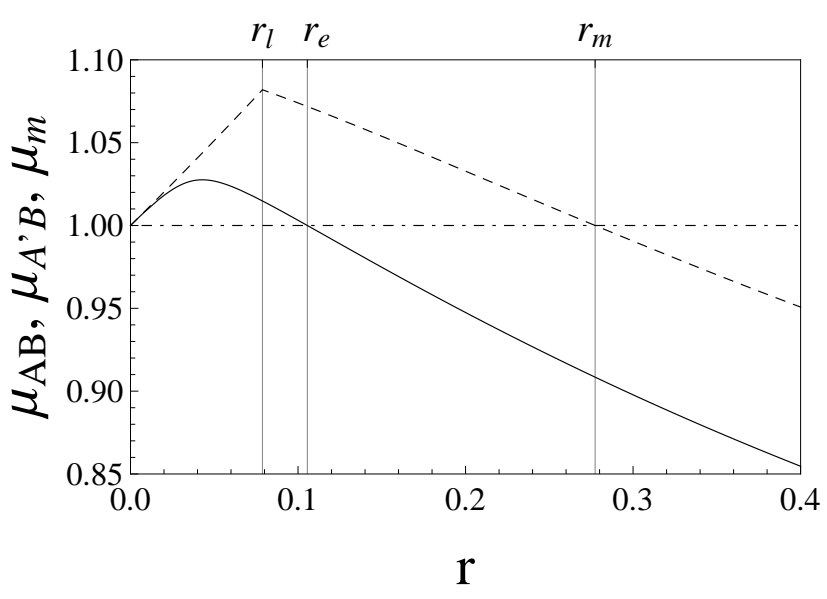

FIG. 2: Lower symplectic eigenvalue $\mu_{A B}=\mu_{A^{\prime} B}$ of the partial transpose of the states with CMs (12) (solid curve) and the symplectic eigenvalue $\mu_{\mathrm{m}}$, Eq. (18), (dashed curve) versus the squeezing parameter $r$ for $\varepsilon=0.1$. The solid vertical lines correspond to the threshold squeezings $r_{1}=0.079$, Eq. (18), $r_{\mathrm{e}}, \mathrm{Eq}$. (14), and $r_{\mathrm{m}}, \mathrm{Eq}$. (20). All the quantities plotted are dimensionless.

$(1 / 2) \ln [2(8 \sqrt{2}-1) / 11] \doteq 0.314$. Thus, for the state with the CM (4) for any $\varepsilon>0$ there exists a region of squeezings $r_{\mathrm{m}} \geq r>r_{\mathrm{e}}$ for which entanglement between Alice and Bob cannot be established by performing any Gaussian measurement on mode $B$ and optimally displacing modes $A$ and $A^{\prime}$ (where one of the modes is held by Bob), but it can be created by superimposing Bob's mode $B$ and the received mode (either $A^{\prime}$ or $A$ ) on a balanced beam splitter.

Note, that the gap exists also in the protocol for the Gaussian entanglement distribution by a separable ancilla [24]. Here, the possibility to localize entanglement between mode $A$ and the transmitted mode $A^{\prime}$ by measurement on Bob's mode $B$ is prevented by separability of mode $A^{\prime}$ from the pair of modes $(A B)$. Nevertheless, a balanced beam splitter on modes $A^{\prime}$ and $B$ creates entanglement between modes $A$ and $B$. Passive coherent unitary operations exhibit superiority over the method based on the measurement and feed-forward also in the two-mode scenario [37]. In this case, the coherent operations allow to extract squeezing from a squeezed signal classically correlated to a probe in cases when the measurement on the probe followed by a feed-forward correction on the signal fails.

\section{DISCUSSION AND CONCLUSION}

The key state of our sharing protocol with the CM (4) is interesting from the point of view of the nondistillable (bound) entanglement [38]. A bipartite quantum state is nondistillable if it is impossible to transform many copies of the state by local operations and classical communication (LOCC) into fewer copies of nearly maximally entan-

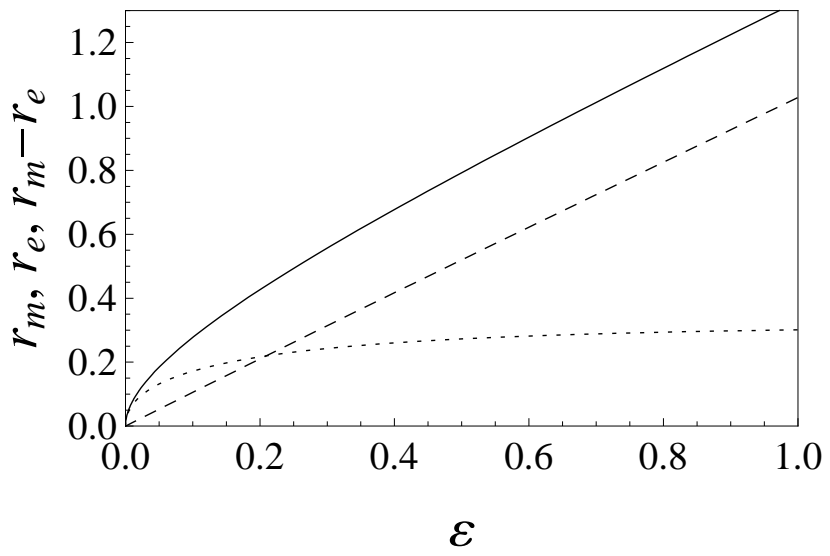

FIG. 3: Squeezing thresholds $r_{\mathrm{m}}$, Eq. (20), (solid curve), $r_{\mathrm{e}}$, Eq. (14), (dashed curve), and their difference $r_{\mathrm{m}}-r_{\mathrm{e}}$ (dotted curve). All the quantities plotted are dimensionless.

gled singlet state. The concept of bound entanglement can be easily generalized to three parties [4] in analogy with the concept of the classical multipartite bound information [6]. We say that a tripartite quantum state is bound entangled if i) any two parties cannot distill singlet states by LOCC even with the help of the third party and ii) the state cannot be created by LOCC. Examples of the tripartite bound entanglement can be found both for two-level systems (qubits) [3, 7] and Gaussian states [15]. They are given by the two-mode biseparable states which are separable across two bipartite splittings and entangled across the third one as well as three-mode biseparable states which are separable across all three bipartite splittings but they are not fully separable. This is because separability across at least two splittings immediately guarantees satisfaction of the condition i) whereas the presence of entanglement causes that also condition ii) holds. However, inseparability with respect to at least two bipartite splittings is currently known to be only a necessary condition for distillability but it is not known whether it is also sufficient. Thus although there are, for instance, distillable one-mode biseparable states both in the qubit [7, 23] as well as Gaussian case [36] one cannot rule out the possibility that there also exist bound entangled states belonging to this class 7, 15]. Our state with the CM (4) is entangled and hence fulfils the condition ii) but moreover it also possesses nontrivial properties which are necessary to satisfy the condition i). First, for any Gaussian state satisfying i) any two parties have to be separable. Namely, if there were entanglement between some pair of parties, then it would be distillable 39]. For our state any two modes are separable and thus no entanglement can be distilled between them if the third party is totally ignored. What is more, not only entanglement cannot be distilled between $A$ and $B$ with the help of $A^{\prime}$ as well as between $A^{\prime}$ and $B$ with the help of $A$ because $B$ is separable from $\left(A A^{\prime}\right)$ but for certain squeezings entanglement also cannot be distilled between $A$ and $A^{\prime}$ 
with the help of Bob provided that he is restricted to the Gaussian measurements on his mode, which is a nontrivial necessary condition for fulfillment of the requirement i).

In conclusion, we have constructed a two-mode separable Gaussian state which can be transformed by splitting of one of its modes on a beam splitter to a state without any two-mode entanglement but with entanglement across two bipartite splittings. If the initial two-mode state is shared by distant Alice and Bob, the beam splitter on Alice's side creates entanglement between one of its outputs and a non-local composite system composed of the other output of the beam splitter and the distant Bob's mode. Although the entanglement is between Alice's mode and the system involving distant Bob's mode it is not entanglement between Alice and Bob because Bob's mode is separable at the same time. The entanglement can nevertheless be turned into entanglement between Alice and Bob by sending one output mode of Alice's beam splitter (which is separable from the other output mode) to Bob and mixing it with Bob's mode on another beam splitter. The protocol just described thus can be interpreted as an entanglement sharing scheme in which entanglement created by Alice's beam splitter can be transformed into entanglement with Bob only if Bob has at his disposal physically also one output mode of the beam splitter. Besides, our analysis shows that for one copy of the key one-mode biseparable state of our protocol entanglement cannot be distilled between any two modes with the help of the third party if Bob is restricted to Gaussian measurements which is a nontrivial property necessary for the presence of the bound entanglement in the state. The question of whether the same holds true also for more generic even non-Gaussian operations on Bob's mode and multiple copies is left for further research. We believe that our results contribute to the better understanding and utilization of entanglement as well as separable correlations in multipartite mixed quantum states.

\section{ACKNOWLEDGMENT}

I would like to thank V. Chille, Ch. Peuntinger, Ch. Marquardt and N. Korolkova for fruitful discussions. The research has been supported by the GACR Project No. P205/12/0694.
[1] D. M. Greenberger, M. Horne, and A. Zeilinger, in Bell's Theorem, Quantum Theory, and Conceptions of the Universe, edited by M. Kafatos (Kluwer, Dordrecht, 1989), p. 69.

[2] J.-W. Pan, D. Bouwmeester, M. Daniell, H. Weinfurter, and A. Zeilinger, Nature (London) 403, 515 (2000).

[3] C. H. Bennett, D. P. DiVincenzo, T. Mor, P. W. Shor, J. A. Smolin, and B. M. Terhal, Phys. Rev. Lett. 82, 5385 (1999).

[4] W. Dür and J. I. Cirac, Phys. Rev. A 62, 022302 (2000).

[5] W. Dür, G. Vidal, and J. I. Cirac, Phys. Rev. A 62, 062314 (2000).

[6] A. Acín, J. I. Cirac and Ll. Masanes, Phys. Rev. Lett. 92, 107903 (2004).

[7] W. Dür, J. I. Cirac, and R. Tarrach, Phys. Rev. Lett. 83, 3562 (1999).

[8] M. Hillery, V. Bužek, and A. Berthiaume, Phys. Rev. A 59, 1829 (1999).

[9] M. Murao, D. Jonathan, M. B. Plenio, and V. Vedral, Phys. Rev. A 59, 156 (1999).

[10] A. Karlsson and M. Bourennane, Phys. Rev. A 58, 4394 (1998).

[11] D. Gottesman and I. L. Chuang, Nature 402, 390 (1999).

[12] M. Zukowski, A. Zeilinger, M. A. Horne, and H. Weinfurter, Acta. Phys. Pol. 93, 187 (1998).

[13] S. L. Braunstein and P. van Loock, Rev. Mod. Phys. 77, 513 (2005).

[14] E. P. Wigner, Phys. Rev. 40, 749 (1932).

[15] G. Giedke, B. Kraus, M. Lewenstein, and J. I. Cirac, Phys. Rev. A 64, 052303 (2001).

[16] P. van Loock and S. L. Braunstein, Phys. Rev. Lett. 84, 3482 (2000).

[17] T. Aoki, N. Takei, H. Yonezawa, K. Wakui, T. Hiraoka, and A. Furusawa, Phys. Rev. Lett. 91, 080404 (2003).

[18] P. van Loock and A. Furusawa, Phys. Rev. A 67, 052315 (2003).

[19] P. van Loock and S. L. Braunstein, Phys. Rev. Lett. 87, 247901 (2001).

[20] H. Yonezawa, T. Aoki, and A. Furusawa, Nature 431, 430 (2004).

[21] T. Tyc and B. C. Sanders, Phys. Rev. A 65, 042310 (2002).

[22] G. Adesso, A. Serafini, and F. Illuminati, Phys. Rev. A 73, 032345 (2006).

[23] T. S. Cubitt, F. Verstraete, W. Dür, and J. I. Cirac, Phys. Rev. Lett. 91, 037902 (2003).

[24] L. Mišta, Jr. and N. Korolkova, Phys. Rev. A 77, 050302 (2008); L. Mišta, Jr. and N. Korolkova, Phys. Rev. A 80, 032310 (2009).

[25] C. E. Vollmer, D. Schulze, T. Eberle, V. Händchen, J. Fiurášek, and R. Schnabel, e-print arXiv:1303.1082 A. Fedrizzi, M. Zuppardo, G. G. Gillett, M. A. Broome, M. de Almeida, M. Paternostro, A. G. White, and T. Paterek, e-print arXiv:1303.4634 Ch. Peuntinger, V. Chille, L. Mišta, Jr., N. Korolkova, M. Förtsch, J. Korger, Ch. Marquardt, and G. Leuchs, e-print arXiv:1304.0504

[26] R. Cleve, D. Gottesman, and H.-K. Lo, Phys. Rev. Lett. 83, 648 (1999).

[27] R. H. Choi, B. Fortescue, and B. C. Sanders, e-print arXiv: 1212.4217

[28] R. Simon, Phys. Rev. Lett. 84, 2726 (2000).

[29] A. Peres, Phys. Rev. Lett. 77, 1413 (1996).

[30] M. Horodecki, P. Horodecki, and R. Horodecki, Phys. Lett. A 223, 1 (1996).

[31] A. Serafini, Phys. Rev. Lett. 96, 110402 (2006); A. Serafini, J. Opt. Soc. Am. B 24, 347 (2007). 
[32] A. Serafini, G. Adesso, and F. Illuminati, Phys. Rev. A 71, 032349 (2005).

[33] M. S. Kim, W. Son, V. Bužek, and P. L. Knight, Phys. Rev. A 65, 032323 (2002).

[34] J. Fiurášek and L. Mišta Jr., Phys. Rev. A 75, 060302 (2007).

[35] G. Vidal and R. F. Werner, Phys. Rev. A 65, 032314 (2002).
[36] L. Mišta, Jr. and J. Fiurášek, Phys. Rev. A 78, 012359 (2008).

[37] R. Filip, Phys. Rev. A 81, 032330 (2010).

[38] M. Horodecki, P. Horodecki, and R. Horodecki, Phys. Rev. Lett. 80, 5239 (1998).

[39] G. Giedke, L.-M. Duan, J. I. Cirac, and P. Zoller, Quant. Inf. Comp. 1, 79 (2001). 